\title{
Comparison between Microplate Spectrometry and LC/MS Chromato- graphy for Facile Pilot Pharmacokinetics and Biodistribution Studies of Doxorubicin-loaded NanoparticleDrug Carriers
}

\author{
Pengxiao Cao and Younsoo Bae* \\ Department of Pharmaceutical Sciences, College of Pharmacy, University of Kentucky, Lexington, KY 40536, USA.
}

\section{ARTICLE INFO \\ Article history: \\ Received on: 07/09/2012 \\ Revised on: 18/09/2012 \\ Accepted on: 22/09/2012 \\ Available online: $28 / 09 / 2012$}

\section{Kay words:}

Drug Delivery,

Polymer Micelles,

Cross-linked Nanoassemblies,

Doxorubicin,

Pharmacokinetics,

Biodistribution

\begin{abstract}
A facile and reliable method to perform pilot pharmacokinetic $(\mathrm{PK})$ and biodistribution studies is necessary for expediting the overall development and clinical translation of novel nanoparticle drug carriers. In this study, we compared two common analytical techniques, fluorescence spectrometry using a microplate reader and liquid chromatography/mass spectrometry (LC/MS), demonstrating the quantification of a model anticancer drug (doxorubicin: DOX) in its free drug and nanoparticle formulations in vivo. Drug-loaded nanoparticle formulations were prepared from poly(ethylene glycol)-poly(aspartate) block copolymers, which formed two model drug carriers with different particle stability, self-assembled polymer micelles (DOX-micelles) and cross-linked nanoassemblies (DOX-CNAs). These three DOX formulations were injected into tumor-bearing mice at a DOX equivalent concentration. DOX levels in liver, spleen, and tumors were found to be comparable regardless of the analytical methods. LC/MS showed lower serum level than spectrometry with a microplate reader, which is consistent with the fact that DOX metabolites are present mainly in the serum. These results demonstrate that, in comparison to the LC/MS method, spectrometry using a microplate reader would be a viable and more facile method to perform pilot PK and biodistribution studies of various potential nanoparticle drug carriers using DOX as a model drug.
\end{abstract}

\section{INTRODUCTION}

Nanoparticle drug carriers, which are typically prepared from biocompatible materials that form vesicular or spherical structures $<200$ nanometers in diameter, have drawn great attention in pharmaceutical sciences for the past decades because they provide novel tools to deliver potent drugs, often promising but poorly-water soluble or too toxic, in controlled manners to specific disease sites in vivo such as cancerous tumors or

* Corresponding Author

Department of Pharmaceutical Sciences, College of Pharmacy,

University of Kentucky, 789 South Limestone, Lexington, KY 40536-0596, USA. Phone: +1-859-323-6649, Fax: +1-859-257-7564 inflammation tissues (Bae et al., 2009; Kim et al., 2010; Blanco et al., 2011). Preparing nanoparticle drug carriers with optimal particle size, stability, and drug release patterns, is generally time-, effort- and cost-consuming as it involves several development steps, such as material synthesis, nanomaterial characterization, in vitro cell assays, and in vivo pharmacokinetics (PK) and biodistribution studies (Ruenraroengsak et al., 2010). Among these steps, the PK and biodistribution studies provide one of the most critical data that lead to 'Go' or 'No-Go' decisions to continue to developa drug carrier. Nevertheless, the PK and biodistribution studies frequently require complicated experimental procedures, such as extraction, 


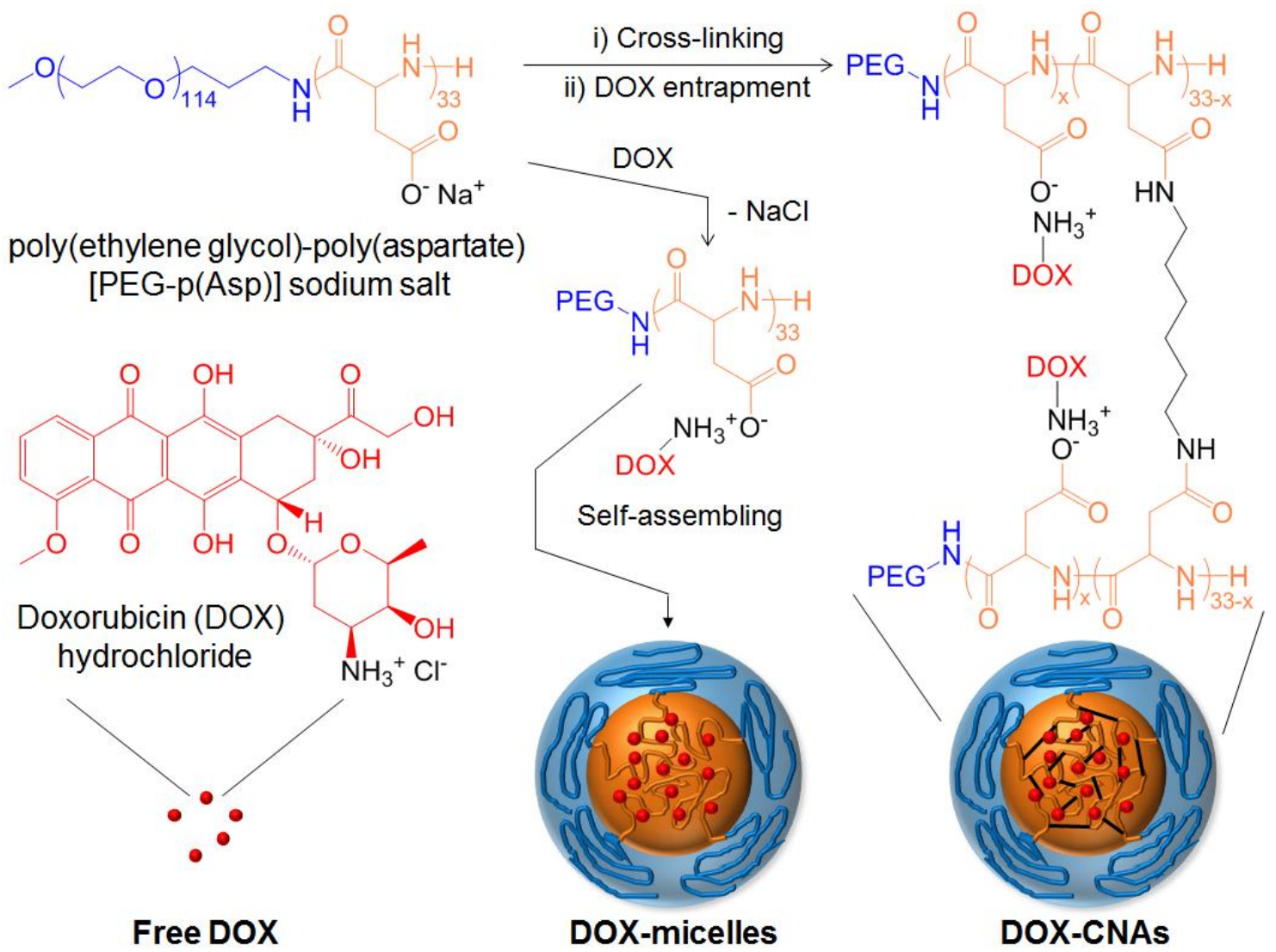

Fig. 1: Drug formulations used in this study. DOX was dissolved in PBS (Free DOX). Block copolymer micelles were prepared from PEG-p(Asp) block copolymers, entrapping DOX inside the micelles through ionic/hydrophobic interactions (DOX-micelles). PEG-p(Asp) block copolymers were cross-linked in the core $(\mathrm{x}=4 \sim 6)$ to prepare a stable nanoparticle drug carrier formulation, followed by DOX entrapment (DOX-CNAs).

isolation, characterization, and data normalization (Gaspar et al., 2009). In addition, instrumental sensitivity and recovery yields of samples from organ tissues as well as blood are often too low to quantify drug payloads released from (or still remained in) drug carriers in vivo. Therefore, there is a critical need to develop a method to perform pilot PK and biodistribution studies for drugloaded nanoparticle carriers in facile and reliable manners, possibly streamlining the overall development process for nanoparticle drug carriers, and thus, bringing promising drug carriers into clinical applications quickly (Duncan et al., 2006). For these reasons, we compared two common analytical instruments, a fluorescence microplate reader and liquid chromatography/mass spectrometer (LC/MS), to design an easy and quick method for performing pilot $\mathrm{PK}$ and biodistribution studies of drug-loaded nanoparticle drug carriers. In this study, we used doxorubicin (DOX) as a model anticancer drug, while preparing model drug-loaded nanoparticles with block copolymer self-assembled micelles (DOX-micelles) and block copolymer cross-linked nano-assemblies (DOX-CNAs) as shown in Figure 1.
DOX is an anthracycline drug, which inhibits cell division by disrupting DNA replication and is widely used for treating various human malignancies such as lymphoma, leukemia, and breast cancer (Muggia et al., 1991; Gewirtz 1999). DOX, red-orange with autofluorescence, is a frequent choice as a model drug because it: 1) is readily detectable by UV-VIS or fluorescence spectrometry; 2) has three functional groups for chemical modification (i.e. amino, hydroxyl, and ketone groups), and most importantly; and 3) shows biological and chemical properties studied well since the discovery in the 1950s. Noticeably, the number of drug delivery studies in which DOX is used as a model drug has increased dramatically: total 1,313 scientific papers with keywords of doxorubicin, adriamycin, and drug delivery were published between 2002 2011 (277 hits in 2011 alone, SciFinder Search). Polymer micelles are nanoparticle drug carriers, $20 \sim 100$ nanometers in diameter, which are generally prepared from selfassembling block copolymers and have a hydrophobic core coated with a hydrophilic shell (Lavasanifar et al., 2002; Torchilin 2010; Miyata et al., 2011). 
Drug payloads can be entrapped in the core of polymer micelles while the hydrophillic shell prevents the drug-loaded polymer micelles from being recognized by the body immune systems. We have been developing polymer micelles for tumor-targeted drug and gene delivery by using biocompatible poly(ethylene glycol)poly(amino acid) block copolymers (Bae et al., 2005; Bae et al., 2009; Lee et al., 2010; Ponta et al., 2010). Our previous studies, including several other clinical trials, have demonstrated that polymer micelles improve water-solubility, blood retention time, tumor-preferential accumulation, and therapeutic efficacy of drug payloads at reduced toxicity (Bae et al., 2009; Matsumura et al., 2009; Plummer et al., 2011; Shin et al., 2011).Meanwhile, crosslinked nanoassemblies (CNAs) are supramolecular assemblies from biocompatible block copolymers that possess drug-binding linkers and cross-linkers in combination, which can be used for controlled drug release maintaining particle size and biocompatibility (Lee et al., 2011; Ponta et al., 2011; Scott et al., 2011). CNAs have a cross-linked core, providing enhanced particle stability in comparison to micelles.

In this study, we demonstrate pilot PK and biodistribution studies, using both microplate reader and LC/MS methods to determine optimal DOX measurement conditions, signal detection limits, and sample extraction yields for quantification of DOX in blood, liver, spleen, and tumors from tumor-bearing mice that received free DOX, DOX-micelles, and DOX-CNAs. Our findings are expected to provide a better understanding of analytical differences between microplate reader and LC/MS methods, which would be useful for early decisionmaking in nanoparticle drug carrier development using DOX as a model drug.

\section{MATERIAL AND METHODS}

\section{Chemicals}

DOX hydrochloride (DOX-HCl), daunorubicin (DAU), 1,6-hexanediamine (HDA), $\beta$-benzyl L-aspartate, triphosgene, dimethyl sulfoxide (DMSO), acetonitrile and formic acid were purchased from Sigma-Aldrich (USA). $\alpha$-Methoxy- $\omega$-amino

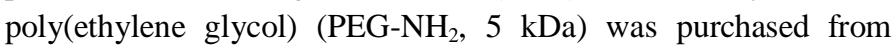
NOF Corporation (Japan). Centrifugal filters $(0.22 \mu \mathrm{m}$ pore size) were purchased from VWR (USA).Regenerated cellulose dialysis bags with molecular weight cut off (MWCO) $6 \sim 8 \mathrm{kDa}$, phosphate buffer solutions, and other lab supplies were purchased from Fisher Scientific (USA).

\section{Cell culture}

A human colon cancer HT29 cell line and McCoy's 5A cell culture medium were obtained from American Type Culture Collection (USA). Fetal bovine serum (FBS) was purchased from Atlanta Biologicals (USA). Hyclone phosphate buffer saline (PBS), trypsin-EDTA (0.25\% trypsin and $2.21 \mathrm{mM}$ EDTA), sterile filters $(0.22 \mu \mathrm{m})$, matrigel, and other cell culture supplies (96-well culture plates, pipettes and flasks) were from Fisher Scientific
(USA). Cells were cultured in media containing $10 \%$ FBS in a humidified atmosphere with $5 \% \mathrm{CO}_{2}$ at $37^{\circ} \mathrm{C}$.

\section{Preparation of nanoparticle drug carriers entrapping DOX}

Biocompatible poly(ethylene glycol)-poly(aspartate) [PEG-p(Asp)] block copolymers were synthesized as reported previously by using $5 \mathrm{kDa}$ PEG and 33 repeating units of aspartate (Bae et al., 2009).As shown in Figure 1, PEG-p(Asp) was used to prepare two types of model nanoparticle drug carriers with different particle stability in vivo: 1) block copolymer selfassembled polymer micelles (micelles) and 2) block copolymer cross-linked nanoassemblies (CNAs) (Lee et al., 2011; Eckman et al., 2012).

The micelles and CNAs are similar in particle size $(<100$ $\mathrm{nm}$ ), but only micelles can dissociate under diluted conditions (e.g. blood in the body). Drug-loaded micelles were prepared by mixing PEG-p(Asp) in a sodium salt form $(10 \mathrm{mg} / \mathrm{mL})$ with DOX-HCl (equivalent to the number of aspartate) in deionized water, which led to DOX entrapment in the micelles through ionic/hydrophobic interactions (Eckman et al., 2012).

CNAs were prepared by cross-linking PEG-p(Asp) with HDA as reported elsewhere (Lee et al., 2011), entrapping DOX$\mathrm{HCl}$ through the same method used for micelles. DOX-loaded nanoparticle drug carriers (DOX-micelles and DOX-CNAs) were freeze-dried and stored at $-20^{\circ} \mathrm{C}$ until use.

\section{DOX fluorescence detection optimization}

We first determined fluorescence intensity of DOX in deionized water by changing DMSO content. DOX was put in water/DMSO mixed solutions $(1.0 \mu \mathrm{g} / \mathrm{ml})$, and its fluorescence was measured time-dependently with $485 \mathrm{~nm}$ excitation and 603 $\mathrm{nm}$ emission wavelengths at which the maximum signal-to-noise $(\mathrm{S} / \mathrm{N})$ ratio can be achieved. By using free DOX as control, relative DOX fluorescence (defined as a relative fluorescence coefficient of DOX) was determined for DOX-micelles and DOXCNAs at $0.5,1,2$, and $4 \mu \mathrm{g} / \mathrm{mL}$ (based on DOX content) in the mixed solutions.

\section{Animal study}

A murine xenograft tumor model was prepared by injecting HT29 cells $\left(2 \times 10^{6}\right.$ cells $)$ in the flank of a six-week old female NCr nude mouse purchased from Taconic (USA). After tumor volume reached approximately $100 \mathrm{~mm}^{3}$, free DOX, DOXmicelles, and DOX-CNAs at $10 \mathrm{mg}$ DOX/kg were injected into mice through a tail vein. Mice received PBS were blank controls. Blood was taken from the mice by cardiac puncture at $0.05,0.5,1$, $3,6,9,12$, and 24 hours post injections $(n=4)$. Blood samples were coagulated and centrifuged to isolate serum. Tumor, liver, and spleen tissues were collected at 6 and $24 \mathrm{~h}$ after euthanization of the mice. Blood and tissue samples were further processed through different methods according to protocols described below for a microplate reader and LC/MS, respectively. 


\section{Microplate reader protocol}

To measure the serum level of DOX and its metabolites, $24 \mu \mathrm{L}$ of serum was mixed with $96 \mu \mathrm{L}$ of DMSO, vortexed and centrifuged at $145,000 \mathrm{rpm}$ for 3 mins. A supernatant $(100 \mu \mathrm{L})$ was taken for fluorescence measurement. Wavelengths were set at $485 \mathrm{~nm}$ and $603 \mathrm{~nm}$ for excitation and emission, respectively.

To measure the tissue level of DOX and its metabolites, a method reported previously to quantify DAU (often used as an internal standard for DOX) was adopted with minor modification (Bachur et al., 1970). Approximately $10 \mathrm{mg}$ of tumor, liver and spleen tissues were mixed with 20 volumes (V/W) of $80 \%$ DMSO and $20 \%$ water, homogenized, and centrifuged. A $100 \mu \mathrm{L}$ supernatant was taken for a fluorescence measurement using a microplate reader (485 $\mathrm{nm}$ excitation, 603 emission).

Calibration curves were established by measuring DOX fluorescence in the serum and tissue samples, which were collected from control mice, mixed with a serial dilution of DOX, and processed as described above. The lowest concentration at which a signal to noise $(\mathrm{S} / \mathrm{N})$ ratio was greater than 3 was defined as the lower limit of quantification.

To estimate ac DOX recovery yield from serum and tissue samples, DOX was prepared in $80 \%$ DMSO and $20 \%$ water at three different concentrations (unprocessed samples) corresponding to those used for the calibration curves in serum and tissue samples (processed samples). The DOX recovery yield was then determined by comparing DOX concentrations in the processed versus unprocessed samples. Measurement precision was expressed as coefficient of variance from repeated sample analyses.

\section{LC/MS protocol}

To measure the serum level of DOX at 6 and $24 \mathrm{~h}$ post sample injections, $50 \mu \mathrm{L}$ of serum was mixed with $10 \mathrm{ng}$ of DAU as an internal standard. Methanol $(700 \mu \mathrm{L})$ was added to the mixture and centrifuged at $145,000 \mathrm{rpm}$ for $10 \mathrm{mins}$. The supernatant was removed and lyophilized. The sample was then resuspended in $100 \mu \mathrm{L}$ of a mobile phase solvent (25\% acetonitrile, $75 \%$ water and $0.1 \%$ formic acid) and centrifuged at $145,000 \mathrm{rpm}$ for 2 mins. The supernatant was then passed through a centrifugal filter $(0.22 \mu \mathrm{m}$ pore size $)$ before analysis using a 6120 Single Quad LC/MS system (Agilent Technologies). Samples were eluted with an Agilent Eclipse XDB-C18 column (4.6 mm $\times 250 \mathrm{~mm}, 5 \mu \mathrm{m})$. A 20 min gradient with water $/ 0.1 \%$ formic acid (solvent $\mathrm{A}$ ) and acetonitrile $/ 0.1 \%$ formic acid (solvent B) at $0.4 \mathrm{~mL} / \mathrm{min}$ was used. The gradient started from $25 \%$ solvent $\mathrm{B}$ and increased to $95 \%$ linearly for 12 mins, then decreased to $25 \%$ solvent B from 12 to 18 mins, maintaining at $25 \%$ solvent $\mathrm{B}$ for another 2 mins. The eluent from LC was delivered to MS via an ESI source. An MS spectrum was acquired in positive mode. DOX and DAU were quantified in selective ion mode (SIM). The total ion mode was used to establish the retention times for DOX and DAU prepared in water initially.

To measure the tissue level of DOX, a liver tissue (35 $\mathrm{mg}$ ) was weighed and spiked with $500 \mathrm{ng}$ of DAU as internal standard. For spleen, $10 \mathrm{mg}$ tissue was spiked with $200 \mathrm{ng}$ of DAU. Then, $700 \mu \mathrm{L}$ of $80 \%$ methanol and $20 \%$ water were added to the tissue, followed by homogenization and centrifugation at $145,000 \mathrm{rpm}$ for $10 \mathrm{mins}$. The supernatant was removed and lyophilized. The sample was then processed following the procedure used for serum sample preparation. The DOX level in a tumor tissue was not investigated using LC/MS due to unsuccessful sample recovery from the tissue as discussed in the Result and Discussion section.

Calibration curves for DOX in serum, liver, and spleen samples were generated by spiking each tissue with a series of known amount of DOX and a fixed amount of DAU as an internal standard: $10 \mathrm{ng}$ of DAU and 80, 40, 20, 10, 5, 2.5, 1.25, 0.63, 0.31, and $0.16 \mathrm{ng}$ of DOX were spiked for blank serum samples; $500 \mathrm{ng}$ of DAU and 4000, 2000, 1000, 500, 250, 125, 62.5, 31.25, 16.63, $7.81 \mathrm{ng}$ of DOX for liver tissues; and $200 \mathrm{ng}$ DAU and 1600, 800, 400, 200, 100, 50, 25, $12.5 \mathrm{ng}$ of DOX for spleen tissues. As described in the microplate reader protocol, DOX recovery yields from serum and other tissues were determined as the ratio of the DOX content in processed samples versus unprocessed samples, while measurement precision was determined by analyzing samples repeatedly and expressed as a coefficient of variance.

\section{PK and statistical analyses}

The serum levels of DOX and its metabolites were measured spectrometrically following the microplate reader and LC/MS protocols. Data were analyzed using Phoenix WinNonlin Software (Version 6.2.1, Pharsight) to obtain pharmacological parameters. All data are expressed mean \pm standard deviation (SD) unless mentioned otherwise. Statistical differences were determined by t-test, one-way, and two-way ANOVA analyses. A difference was considered statistically significant when $p<0.05$.

\section{RESULTS AND DISCUSSION}

\section{Preparation of DOX-loaded nanoparticle drug carriers}

Dynamic light scattering (DLS) measurements showed that the average particle sizes of DOX-micelles and DOX-CNAs were 61.6 and $56.5 \mathrm{~nm}$, respectively (Figure 2). Both DOX-loaded nanoparticle drug carriers were stable in the absence of counter ions (deionized water) showing no drug precipitates. However, water-insoluble debris was seen when DOX-micelles were reconstituted from a freeze-dried powder. On the contrary, DOXCNAs were readily soluble in water. For these reasons, we prepared DOX-micelles and CNAs freshly in deionized water, diluted the nanoparticle carriers with buffer solutions, and removed all impurities with $0.22 \mu \mathrm{m}$ filters prior to each experiment.

\section{DOX fluorescence detection optimization}

DOX fluorescence in water and DMSO mixed solutions was clearly dependent on DMSO concentration ( $p<0.001)$ as shown in Figure 3. We confirmed that DOX showed the strongest fluorescence signal with the least variation between nanoparticle 
carriers when it is quantified in a mixed solution from $80 \%$ DMSO and $20 \%$ water. The maximum DOX fluorescence intensity at $80 \%$ DMSO was used to normalize percent relative fluorescence intensity (RFI\%) for other conditions. DOX in $100 \%$ water showed $38.2 \pm 2.1$ RFI\% while DOX in $100 \%$ DMSO yielded 66.0 $\pm 0.7 \mathrm{RFI} \%$. DOX fluorescence remained stable in $80 \%$ DMSO over 90 minutes, which is long enough to process in vivo samples and take measurements to quantify signals. In other mixed solutions, DOX fluorescence signals changed over time. There was no significant difference in DOX fluorescence intensity among free DOX (100 RFI\%), DOX-micelles $(105.7 \pm 11.5 \mathrm{RFI} \%)$, and DOX-CNAs $(95.1 \% \pm 8.5 \mathrm{RFI} \%)$ with $\mathrm{p}>0.05$. We previously observed a fluorescence quenching phenomenon for DOX entrapped in the micelles in aqueous solutions, which is attributed to high DOX concentrations elevated locally and DOX molecules tightly packed in the micelle core (Bae et al., 2003), but in this study, 80\% DMSO seemed to break down the micelles to avoid DOX fluorescence quenching. Interestingly, CNAs also showed no DOX fluorescence quenching in $80 \%$ DMSO, indicating that DOX molecules in CNAs are not tightly packed in this particle formulation, although further physicochemical characterization of these distinctive nanoparticle drug carriers is necessary in future studies.

\section{DOX detection from blood and organ tissues}

DOX levels in serum, liver and spleen were determined for free DOX and DOX-micelles at 6 and $24 \mathrm{~h}$ as shown in Table 1 using the microplate reader and LC/MS methods. The retention times of DOX and DAU in LC/MS spectrum were approximately 7.7 and 8.4 minutes respectively (Figure 4). When samples were processed through the microplate reader protocol, DOX recovery yields from serum, liver, spleen, and tumor samples were $96.3 \pm$ $4.6 \%, 79.9 \pm 3.5 \%, 74.8 \pm 6.9 \%$ and $80.2 \pm 5.5 \%$, while DOX detection limits were $0.02 \mu \mathrm{g} / \mathrm{ml}, 0.31 \mu \mathrm{g} / \mathrm{g}, 0.25 \mu \mathrm{g} / \mathrm{g}$, and $0.25 \mu \mathrm{g} / \mathrm{g}$, respectively. One of the issues to trouble accurate drug quantification from in vivo samples is production of drug metabolites in vivo. DOX consists of four cycline rings on its anthracycline structure, conjugated with an amino sugar (Figure 1). DOX undergoes carbonyl reduction and deglycosidation in vivo, resulting in several metabolites, such as doxorubicinol, doxorubicinone, or doxorubicinolone, although doxorubicinol is the major bioactive metabolite (Beijnen et al., 1991; Ferrazzi et al., 1991; Maudens et al., 2011). These metabolites are also fluorescent due to the similar tetracycline structures with similar fluorescence intensities in comparison to DOX. DOX metabolites exist in tissues at low levels, but relatively abundant in plasma (Peters et al., 1981; van Asperen et al., 1998). Therefore, capability of measuring DOX and its metabolites simultaneously is one of the advantages that the microplate reader method can provide. The protocol for LC/MS resulted in $65.7 \pm 5.1 \%, 54.1 \pm 6.8 \%$ and $58.2 \pm 8.7 \%$ DOX recovery yields from serum, liver, and spleen samples, while the DOX detection limits were $0.0032 \mu \mathrm{g} / \mathrm{ml}, 0.22 \mu \mathrm{g} / \mathrm{g}$, and 0.25 $\mu \mathrm{g} / \mathrm{g}$, respectively. It must be noted that DOX extraction for LC/MS analysis of DOX-CNAs was unsuccessful, demonstrating that the quality of LC/MS data is heavily dependent on the sample preparation step. Incomplete DOX release from CNAs, even in the presence of organic solvent (methanol), would be also problematic to extract a sufficient amount of DOX for LC/MS analyses. In comparison to the microplate reader method that detects DOX and its metabolites directly, the LC/MS method requires complicated sample preparation procedures, although free DOX and the internal standard (DAU) were collected at relatively high yields (Figure 4). Despite the difference in technical difficulty and measurement sensitivity, both microplate reader and LC/MS methods presented similar results in terms of determining DOX levels in in vivo samples ( $\mathrm{p}>0.05$ ). However, the LC/MS analysis always showed lower DOX levels (approximately $12.2 \sim 23.8 \%$ ) in the liver and spleen samples in comparison to the microplate reader method. For serum samples, DOX levels determined by the LC/MS method were $29.1 \sim 53.9 \%$ of those determined by the microplate reader method, indicating that LC/MS detects DOX alone while a microplate reader includes signals from DOX and its metabolites. These results are consistent with the fact that DOX metabolites exist mainly in the serum but not tissues (Beijnen et al., 1991; Ferrazzi et al., 1991; Maudens et al., 2011).
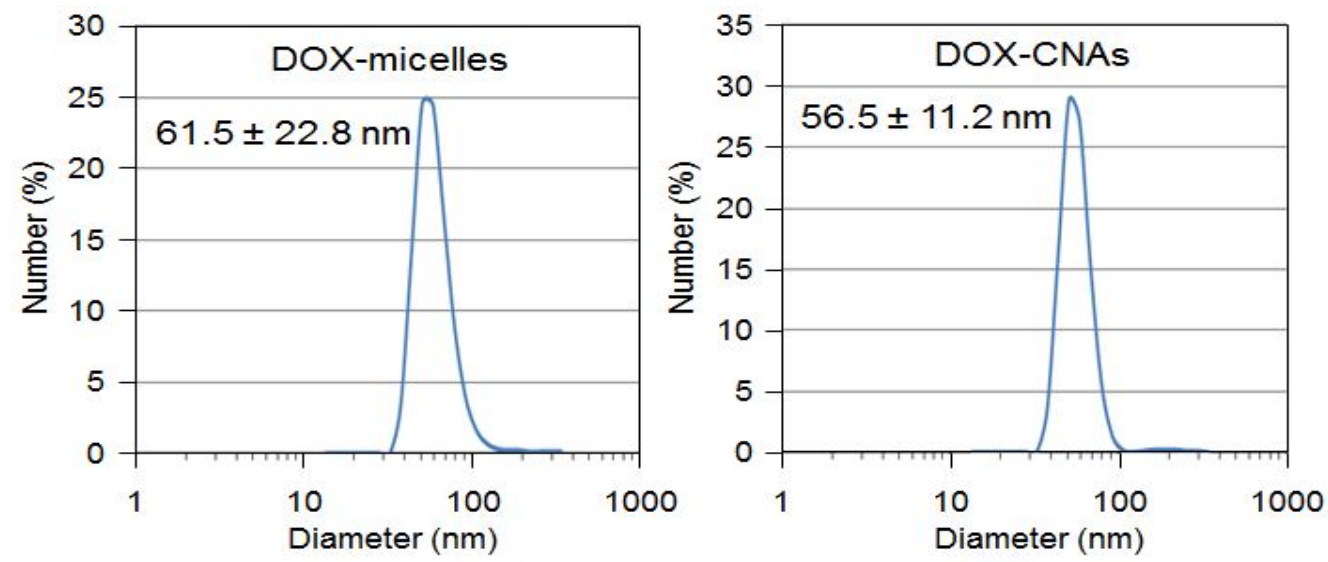

Fig. 2: Particle size distribution determined by dynamic light scattering (DLS) measurements. 


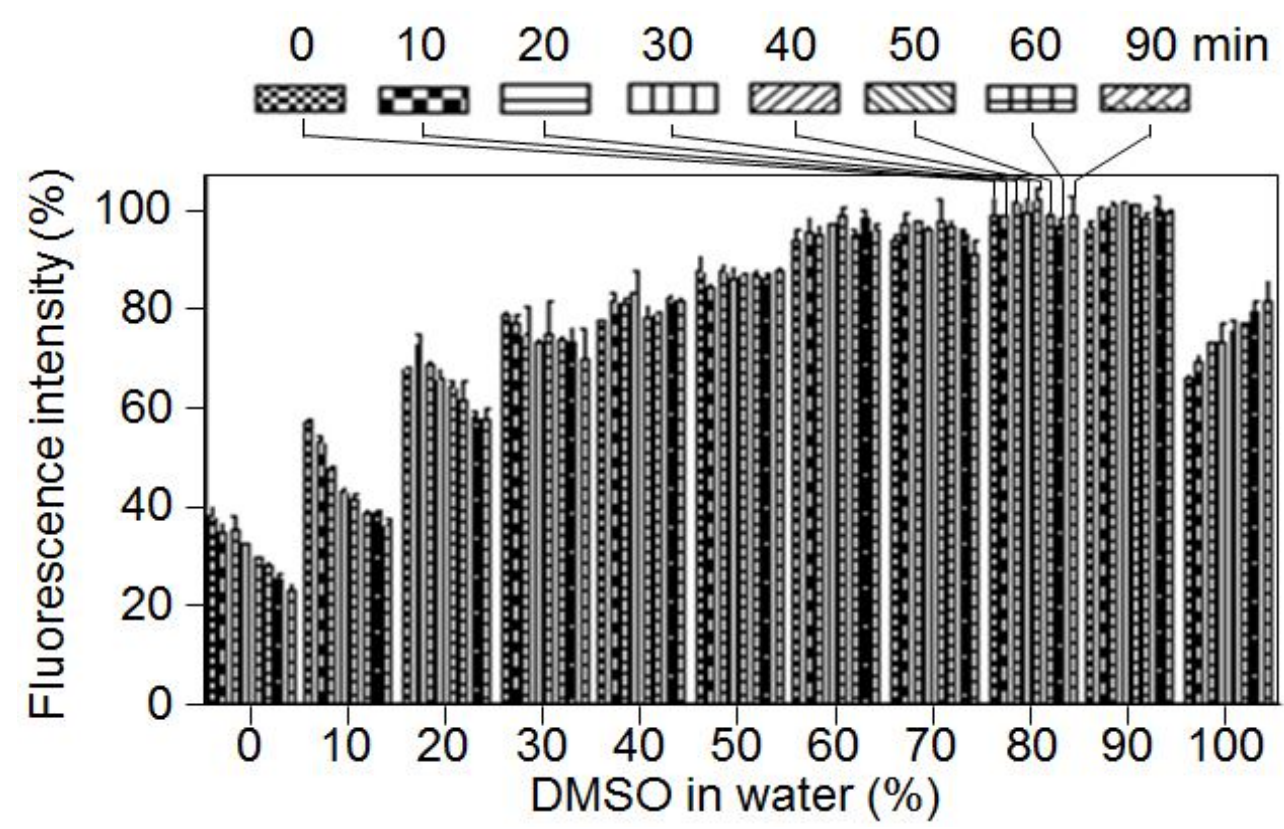

Fig. 3: Fluorescence intensity of DOX at various \%DMSO/water. DOX fluorescence was measured time-dependently with $485 \mathrm{~nm}$ excitation and $603 \mathrm{~nm}$ emission wavelengths.
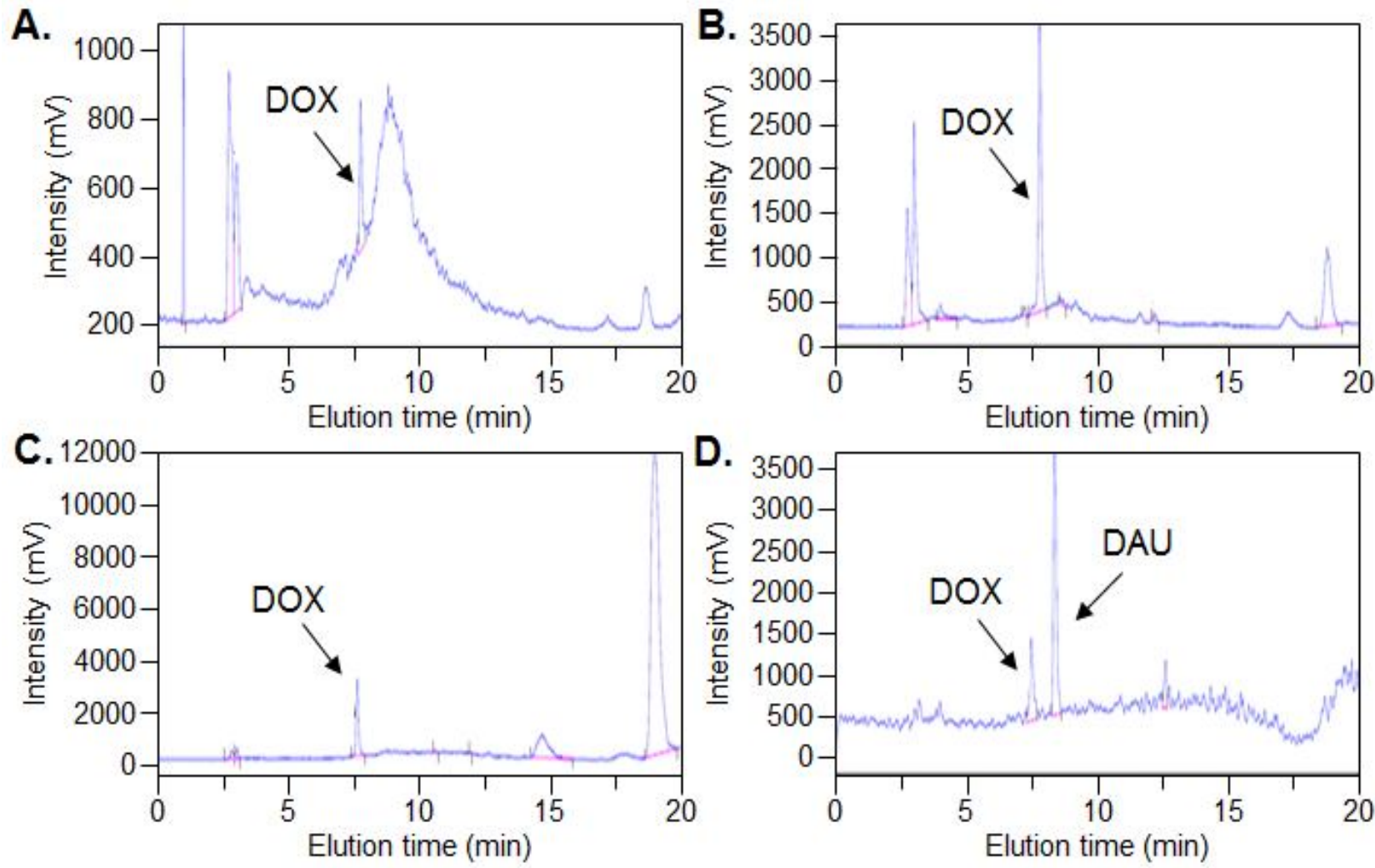

Fig. 4: Representative LC/MS spectra.(A) Representative LC/MS spectrum of serum sample in selective ion mode (SIM).(B) Representative LC/MS spectrum of liver sample (SIM). (C) Representative LC/MS spectrum of spleen sample (SIM). (D) Separation of DOX from DAU internal standard in total ion mode (TIM). 


\section{Pilot pharmacokinetics and biodistribution studies}

Our data demonstrate that spectrophotometry using a microplate reader would be a convenient and viable method to quantify DOX because it requires a reasonably small amount of sample $(20 \mu \mathrm{L}$ serum and $10 \mathrm{mg}$ of tissues) and the sample preparation is time- and cost-effective, although it cannot differentiate DOX from its metabolites. In comparison, methods to quantify DOX in serum and tissues generally require UV-VIS/FL spectrophotometry and high-performance liquid chromatography (HPLC), which detect the autofluorescence of DOX and its metabolites (Yesair et al., 1972; Maudens et al., 2011). HPLC with a fluorescence detector has been a method of choice for many years, and more recently LC/MS and LC/MS/MS provide analytical methods that lower detection limits (Maudens et al., 2011).

To compare the microplate reader and LC/MS methods further, we performed pilot pharmacokinetics and biodistribution studies, injecting free DOX, DOX-micelles, and DOX-CNAs into tumor-bearing mice. As shown in Tables 1 and 2, statistical analyses showed a significant difference of serum concentration of DOX among samples ( $\mathrm{p}<0.001)$. The serum DOX concentrationtime curves were biphasic $(\alpha, \beta)$, showing an initial distribution phase and subsequent elimination phase (Figure 5). The biphasic pattern was further described by a two-compartment model as the pharmacokinetic parameters are listed in Table 2. DOX clearance was $64.94 \pm 21.78,27.06 \pm 0.97$, and $4.51 \pm 0.79 \mathrm{~mL} / \mathrm{h}$ for free drug, micelle, and CNA formulations, respectively. The area under the serum concentration-time curve (AUC) demonstrates that nanoparticle drug carriers maintained DOX concentrations in the blood for a prolonged time in comparison to free drug, and the CNA formulation showed the largest AUC $(44.30 \pm 7.74 \mathrm{~h}$ $\mu \mathrm{g} / \mathrm{mL})$.

These results demonstrate that the microplate reader method is comparable to the LC/MS method. The microplate reader method appeared to be beneficial particularly in simplifying sample preparation procedures. The sample preparation procedure involves multiple steps for the LC/MS method, resulting in a sample loss and lower DOX recovery yield. In addition, DOX collected from the tissue through extraction does not include DOX still entrapped inside nanoparticle drug carriers, which make the detection method problemic (DOX-CNAs in this study). The microplate reader method allows the detection of DOX released and still entrapped in nanoparticle drug carriers, which showed similar RFI\%, and thus providing more meaningful DOX concentrations in in vivo samples.

Based on these results, we finally determined DOX levels in liver, spleen, and tumor tissues using the microplate reader method (Figure 6). LC/MS analyses could not be performed due to the limitation of DOX recovery from the nanoparticle drug carriers accumulated in tissues as discussed above. The results revealed that the CNA formulation significantlyincreased DOX accumulation in tumor tissue $(5.42 \pm 1.14 \mu \mathrm{g} / \mathrm{g})$ in comparison to free $\operatorname{DOX}(2.51 \pm 1.55 \mu \mathrm{g} / \mathrm{g})$ at $24 \mathrm{~h}$ post drug administration, while the micelle formulation showed no significant difference $(3.69 \pm 0.80 \mu \mathrm{g} / \mathrm{g})$.

It is noted that dramatic liver accumulation of DOX was also found in CNA and micelle formulations $(32.2 \pm 9.28 \mu \mathrm{g} / \mathrm{g}$, $8.70 \pm 7.16 \mu \mathrm{g} / \mathrm{g}$ vs $3.17 \pm 0.96 \mu \mathrm{g} / \mathrm{g}$ in free drug formulation), suggesting that further optimization of these two model drug carrier formulations would be necessary. Nevertheless, this study demonstrates that the microplate reader method is useful to perform pilot PK and biodistribution studies using DOX as a model drug in facile and reliable manners to provide a better understanding of drug carrier performance in vivo.

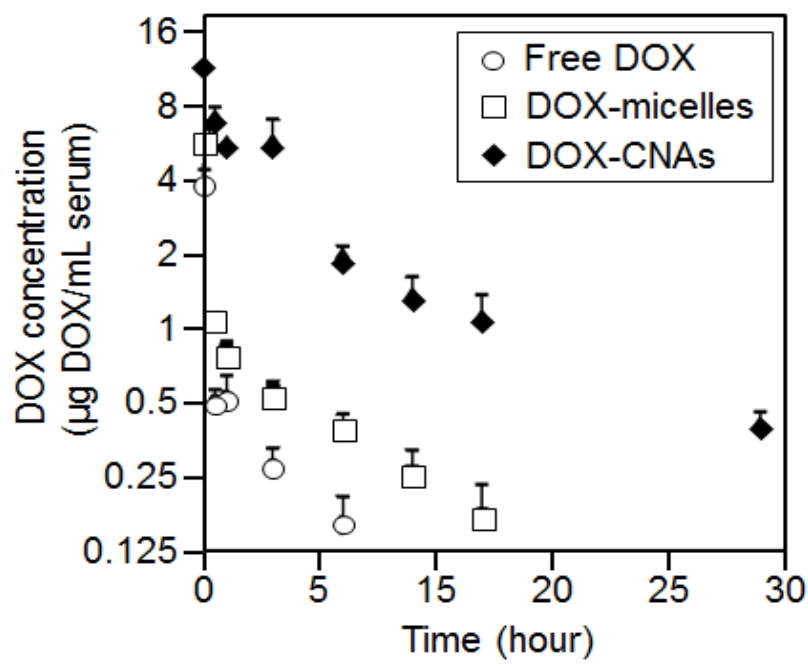

Fig. 5: Serum concentrations of DOX equivalents in nude mice treated with free DOX, DOX-micelles, and DOX-CNAs.

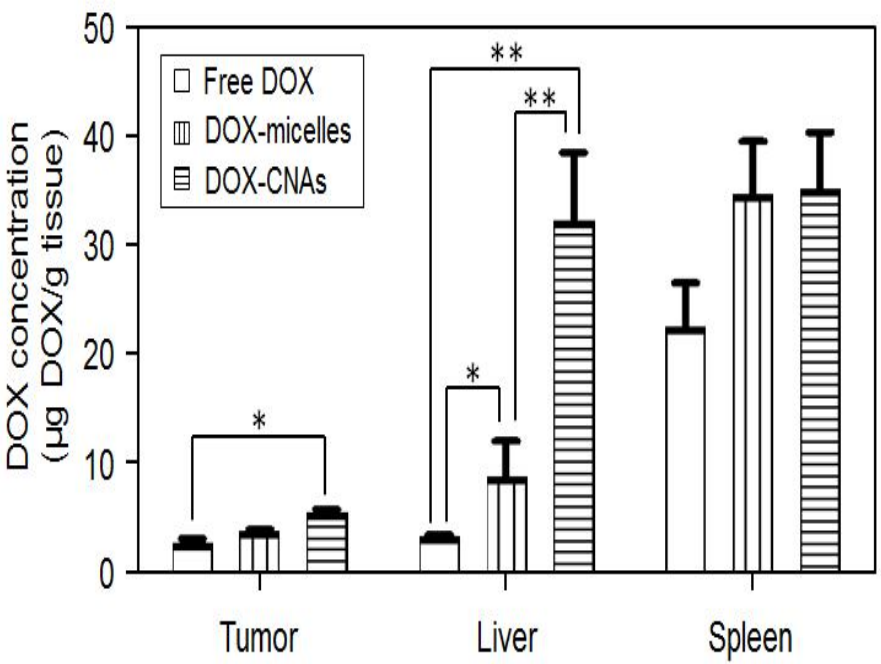

Fig. 6: DOX concentration in liver, spleen, and tumor tissues $24 \mathrm{~h}$ after systemic injection of drug formulations. Data were expressed as means $\pm \mathrm{SD}$ $(* \mathrm{p}<0.05, * * \mathrm{p}<0.01)$. 
Table. 1: Comparison of the microplate reader and LC/MS methods to determine DOX levels in vivo.

\begin{tabular}{|c|c|c|c|c|c|}
\hline & & \multicolumn{2}{|l|}{ Free DOX } & \multicolumn{2}{|c|}{ DOX-Micelle } \\
\hline & & $6 \mathrm{~h}$ & $24 \mathrm{~h}$ & $6 \mathrm{~h}$ & $24 \mathrm{~h}$ \\
\hline \multirow{3}{*}{$\begin{array}{l}\text { Microplate reader method } \\
(\text { mean } \pm \text { SD })\end{array}$} & Serum* & $0.2 \pm 0.1$ & - & $0.4 \pm 0.1$ & - \\
\hline & Liver** & $15.2 \pm 5.5$ & $3.2 \pm 1.0$ & $33.9 \pm 5.1$ & $8.7 \pm 7.2$ \\
\hline & Spleen** & $16.4 \pm 3.6$ & $22.4 \pm 8.7$ & $25.4 \pm 7.3$ & $34.7 \pm 10.3$ \\
\hline \multirow{3}{*}{$\begin{array}{l}\text { LC/MS method } \\
(\text { mean } \pm \mathrm{SD})\end{array}$} & Serum* & $0.09 \pm 0.03$ & $0.03 \pm 0.01$ & $0.1 \pm 0.02$ & $0.05 \pm 0.02$ \\
\hline & Liver** & $12.5 \pm 1.9$ & $2.8 \pm 2.1$ & $26.6 \pm 5.5$ & $7.3 \pm 4.0$ \\
\hline & Spleen** & $14.4 \pm 2.9$ & $17.1 \pm 6.0$ & $21.9 \pm 2.2$ & $27.8 \pm 2.6$ \\
\hline \multirow{3}{*}{$\begin{array}{l}\text { Relative intensity of LC/MS } \\
(\%)\end{array}$} & Serum* & 53.9 & - & $29.1 * * *$ & - \\
\hline & Liver** & 82.2 & 87.5 & 78.5 & 83.9 \\
\hline & Spleen** & 87.8 & 76.2 & 86.1 & 80.2 \\
\hline
\end{tabular}

Table. 2: Pharmacokinetic parameters using a two-compartment model.

\begin{tabular}{|c|c|c|c|}
\hline & Free DOX & DOX-micelles & DOX-CNAs \\
\hline $\mathrm{AUC}^{*}(\mathrm{~h} \mu \mathrm{g} / \mathrm{mL})$ & $3.08 \pm 1.03$ & $7.39 \pm 0.26$ & $44.30 \pm 7.74$ \\
\hline CL** (mL/hour) & $64.94 \pm 21.78$ & $27.06 \pm 0.97$ & $4.51 \pm 0.79$ \\
\hline $\mathrm{t}_{1 / 2}(\alpha)(\mathrm{h})$ & $0.04 \pm 0.63$ & $0.10 \pm 0.00$ & $0.12 \pm 0.18$ \\
\hline $\mathrm{t}_{1 / 2}(\beta)(\mathrm{h})$ & $3.17 \pm 1.25$ & $4.93 \pm 0.29$ & $4.18 \pm 1.09$ \\
\hline
\end{tabular}

*AUC: The area under the serum concentration-time curve; **CL: clearance

\section{CONCLUSION}

In this study, we have demonstrated that spectrophotometry using a microplate reader is as effective as LC/MS to perform pilot pharmacokinetics and biodistribution studies for model nanoparticle drug carriers entrapping an anticancer drug DOX.We compared a microplate reader and LC/MS to determine DOX levels in the serum and tissues, following intravenous injections of free drug and model nanoparticle drug carriers (DOX-micelles and DOX-CNAs). Spectrophotometry using a microplate reader was only able to detect combined signals from DOX and its metabolites while LC/MS differentiated DOX accurately. LC/MS is more sensitive in term of determining DOX concentrations in the serum than a microplate reader. However, DOX concentrations in tissues were similar regardless of the analytical method, suggesting that the amounts of DOX metabolites are very limited in tissues. Interestingly, DOX concentrations in the serum measured is approximately $40 \sim 70 \%$ greater with the microplate reader method than the LC/MS method, indicating that more DOX metabolites exist in serum. The excitation and emission wavelengths of DOX are optimized at 485 and $603 \mathrm{~nm}$ in this study as they yielded the highest $\mathrm{S} / \mathrm{N}$ ratio and few naturally occurring compounds show fluorescence at these wavelengths. Therefore, the results in this study conclude that, as far as DOX is used as a model drug, the microplate reader method is: 1) time- and cost-saving; 2) viable for limited amounts of samples (approximately $20 \mu \mathrm{L}$ serum and 10 mg tissues were used in this study); 3) simple in preparing samples with higher recovery yields from blood and in vivo tissues; 4) highly precise ( $<2 \%$ of variance between samples) comparing with LC/MS (3 15\% variance); and 5) capable of measuring both DOX and its metabolites at the same time. Our findings are expected to contribute to facilitating PK and biodistribution studies critical for making decisions on the development of various types of nanoparticle drug carriers in biomedical and pharmaceutical applications.

\section{ACKNOWLEDGMENTS}

Authors acknowledge financial support provided by the Kentucky Lung Cancer Research Program.

\section{REFERENCES}

A. Lavasanifar, J. Samuel, and G.S. Kwon. Poly(ethylene oxide)-block-poly(L-amino acid) micelles for drug delivery. Adv Drug Deliv Rev 2002; 2: 169-190.

A.M. Eckman, E. Tsakalozou, N.Y. Kang, A. Ponta, and Y. Bae. Drug Release Patterns and Cytotoxicity of PEG-poly(aspartate) Block Copolymer Micelles in Cancer Cells. Pharm Res 2012; 7: 17551767.

A. Ponta, S. Akter, and Y. Bae. Degradable cross-linked nanoassemblies as drug carriers for heat shock protein 90 inhibitor 17-Nallylamino-17-demethoxygeldanamycin. Pharmaceuticals 2011; 12811292.

A. Ponta, and Y. Bae. PEG-poly(amino acid) block copolymer micelles for tunable drug release. Pharm Res 2010; 11: 2330-2342.

B.Y. Kim, J.T. Rutka, and W.C. Chan. Nanomedicine. $N$ Engl J Med 2010; 25: 2434-2443.

D.A. Gewirtz. A critical evaluation of the mechanisms of action proposed for the antitumor effects of the anthracycline antibiotics adriamycin and daunorubicin. Biochem Pharmacol 1999; 7: 727-741.

D. Scott, J. Rohr, and Y. Bae. Nanoparticulate formulations of mithramycin analogs for enhanced cytotoxicity. Int J Nanomed 2011; 2757-2767.

D.W. Yesair, E. Schwartzbach, D. Shuck, E.P. Denine, and M.A. Asbell. Comparative pharmacokinetics of daunomycin and adriamycin in several animal species. Cancer Res 1972; 6: 1177-1183.

E. Blanco, A. Hsiao, A.P. Mann, M.G. Landry, F. MericBernstam, and M. Ferrari. Nanomedicine in cancer therapy: innovative trends and prospects. Cancer Sci 2011; 7: 1247-1252.

E. Ferrazzi, J.M. Woynarowski, A. Arakali, D.E. Brenner, and T.A. Beerman. DNA damage and cytotoxicity induced by metabolites of anthracycline antibiotics, doxorubicin and idarubicin. Cancer Commun 1991; 6: 173-180.

F.M. Muggia, and M.D. Green. New anthracycline antitumor antibiotics. Crit Rev Oncol Hematol 1991; 1: 43-64. 
H.C. Shin, A.W. Alani, H. Cho, Y. Bae, J.M. Kolesar, and G.S. Kwon. A 3-in-1 polymeric micelle nanocontainer for poorly water-soluble drugs. Mol Pharm 2011; 4: 1257-1265.

H.J. Lee, and Y. Bae. Cross-Linked Nanoassemblies from Poly(ethylene glycol)-poly(aspartate) Block Copolymers as Stable Supramolecular Templates for Particulate Drug Delivery. Biomacromolecules 2011.

H.J. Lee, A. Ponta, and Y. Bae. Polymer nanoassemblies for cancer treatment and imaging. Ther Delivery 2010; 6: 803-817.

J.H. Peters, G.R. Gordon, D. Kashiwase, and E.M. Acton. Tissue distribution of doxorubicin and doxorubicinol in rats receiving multiple doses of doxorubicin. Cancer Chemother Pharmacol 1981; 1: 6569.

J. Van Asperen, O. van Tellingen, and J.H. Beijnen. Determination of doxorubicin and metabolites in murine specimens by high-performance liquid chromatography. J Chromatogr B Biomed Sci Appl 1998; 1-2: 129-143.

J.H. Beijnen, P.L. Meenhorst, R. van Gijn, M. Fromme, H. Rosing, and W.J. Underberg. HPLC determination of doxorubicin, doxorubicinol and four aglycone metabolites in plasma of AIDS patients. $J$ Pharm Biomed Anal 1991; 10-12: 995-1002.

K.E. Maudens, C.P. Stove, and W.E. Lambert. Quantitative liquid chromatographic analysis of anthracyclines in biological fluids. $J$ Chromatogr B Analyt Technol Biomed Life Sci 2011; 25: 2471-2486.

K. Miyata, R.J. Christie, and K. Kataoka. Polymeric micelles for nano-scale drug delivery. React Funct Polym 2011; 3: 227-234.

P. Ruenraroengsak, J.M. Cook, and A.T. Florence. Nanosystem drug targeting: Facing up to complex realities. J Control Release 2010; 3: 265-276.

R. Gaspar, and R. Duncan. Polymeric carriers: preclinical safety and the regulatory implications for design and development of polymer therapeutics. Adv Drug Deliver Rev 2009; 13: 1220-1231.
R. Duncan, H. Ringsdorf, and R. Satchi-Fainaro. Polymer therapeutics: polymers as drugs, drug and protein conjugates and gene delivery systems: past, present and future opportunities. Advances in Polymer Science 2006; Polymer Therapeutics I: 1-8.

R. Plummer, R.H. Wilson, H. Calvert, A.V. Boddy, M. Griffin, J. Sludden, M.J. Tilby, M. Eatock, D.G. Pearson, C.J. Ottley, Y. Matsumura, K. Kataoka, and T. Nishiya. A Phase I clinical study of cisplatin-incorporated polymeric micelles (NC-6004) in patients with solid tumours. Br J Cancer 2011; 4: 593-598.

V.P. Torchilin. Polymeric micelles for therapeutic applications in medicine. RSC Nanoscience \& Nanotechnology (Polymer-Based Nanostructures: Medical Applications) 2010; 261-299.

Y. Bae, and K. Kataoka. Intelligent polymeric micelles from functional poly(ethylene glycol)-poly(amino acid) block copolymers. $A d v$ Drug Deliv Rev 2009; 10: 768-784.

Y. Bae, S. Fukushima, A. Harada, and K. Kataoka. Design of environment-sensitive supramolecular assemblies for intracellular drug delivery: Polymeric micelles that are responsive to intracellular $\mathrm{pH}$ change. Angew Chem Int Ed 2003; 38: 4640-4643.

Y. Bae, W.D. Jang, N. Nishiyama, S. Fukushima, and K. Kataoka. Multifunctional polymeric micelles with folate-mediated cancer cell targeting and $\mathrm{pH}$-triggered drug releasing properties for active intracellular drug delivery. Mol Biosyst 2005; 3: 242-250.

Y. Matsumura, and K. Kataoka. Preclinical and clinical studies of anticancer agent-incorporating polymer micelles. Cancer Sci 2009; 4: $572-579$

\section{How to cite this article:}

Pengxiao Cao and Younsoo Bae. Comparison between Microplate Spectrometry and LC/MS Chromato-graphy for Facile Pilot Pharmacokinetics and Biodistribution Studies of Doxorubicinloaded Nanoparticle Drug Carriers. J App Pharm Sci. 2012; 2(9): 001-009. 